\title{
A Case of Infantile Kyrle-Flegel Disease in a 6-Year-Old Yemeni Girl
}

\author{
Mohammad Ali Alshami Mona Jameel Mohana \\ Dermatology Department, University Hospital, Sana'a University, Sana'a, Yemen
}

\section{Key Words}

Kyrle disease $\cdot$ Flegel disease $\cdot$ Papule $\cdot$ Nodule $\cdot$ Keratotic plug

\begin{abstract}
Kyrle disease (KD) and Flegel disease (FD) are rare variants of primary perforating dermatoses, characterized by transepidermal elimination of abnormal endogenous materials. We describe a 6-year-old Yemeni girl with a 2-year history of generalized asymptomatic, small, reddish-brown keratotic papules with a lenticular central keratotic plug. Although these features are synonymous with FD, the histology of a punch biopsy was consistent with KD. The patient was otherwise healthy, and no family members had a history of similar diseases. The patient was diagnosed with KD-FD, owing to the manifestation of features associated with both diseases.

(C) 2016 The Author(s) Published by S. Karger AG, Basel
\end{abstract}

\section{Introduction}

In 1916, the Austrian dermatologist Josef Kyrle described multiple hyperpigmented papules and nodules 5-10 $\mathrm{mm}$ in size with a central keratotic plug in a 22-year-old diabetic woman. He termed this condition 'hyperkeratosis follicularis et parafollicularis in cutem penetrans' because it sometimes involved the follicular (follicularis) or interfollicular (parafollicularis) epidermis, and in most cases, the keratinous plug penetrated into the dermis (penetrans) [1]. Early lesions consisted of minute, slightly elevated follicular papules resembling those seen in keratosis pilaris. Older lesions were bean-sized, elevated bright brown papules with central scale [2]. Since the initial report, hundreds of cases of Kyrle disease (KD), as it is now known, have been described worldwide, $10 \%$ of which involved chronic renal failure and hemodialysis (emedicine.medscape.com/article/1074469). In most reported cases, KD was acquired and associated with other systemic diseases such as diabetes mellitus. In those cases in which it was primary and not associated with other systemic dis-

\section{KARGER}

M. Alshami, MD

Dermatology Department, University Hospital, Sana'a University

PO Box 1950

Sana'a (Yemen)

E-Mail mohammadalshami62@gmail.com 
Alshami and Mohana: A Case of Infantile Kyrle-Flegel Disease in a 6-Year-Old Yemeni Girl

eases [3], it was familial in a few instances [4, 5]. Therefore, KD appears to be a rare genodermatosis with both autosomal dominant and recessive inheritance. KD is considered to be one of the perforating dermatoses (more precisely referred to as 'dermatoses with transepidermal elimination'), which include perforating collagenosis, elastosis perforans serpiginosa, and perforating folliculitis.

In 1958, the German dermatologist Heinz Flegel described a case involving generalized keratotic papules 1-5 $\mathrm{mm}$ in size [6]. The pathology typically included discrete hyperkeratosis or parakeratosis along with epidermal atrophy and lichenoid infiltrates. He termed this condition 'hyperkeratosis lenticularis perstans', owing to the persistent (perstans) presence of a disc-like central keratinous plug (lenticularis). Since his report, there has been no agreement as to whether this condition is a discrete entity or a KD variant $[7,8]$. Flegel disease (FD), as it is now known, is considered a genodermatosis with autosomal dominant inheritance, manifesting in the third to fourth decade of life, although it has been reported in patients as young as 13 years of age [9].

There are very few reports of patients who exhibit the signs of both diseases, collectively termed 'KD-FD' [4, 10]. We report a rare case of infantile KD-FD in a 6-year-old Yemeni girl who presented with the clinical manifestations of FD and the histology of KD.

\section{Case Presentation}

A 6-year-old Yemeni girl presented with a 2-year history of generalized asymptomatic papules with a central lentiform keratinous plug (fig. 1). She was otherwise healthy as indicated by a clinical examination and laboratory tests (complete blood counts, renal function test, and fasting blood sugar measurement). There was no history of skin conditions among her siblings or parents.

The patient exhibited the clinical characteristics of FD; i.e., generalized small lesions (1$5 \mathrm{~mm}$, fig. 1a) mainly over the face, trunk, and extensor surfaces of the extremities, rather than the large lesions typically observed in KD (5-15 mm). Closer examination revealed that the lesions were at different stages of development, namely follicular or parafollicular (fig. 1b). A hair protruded from some of the follicular lesions but not from the parafollicular lesions. Lesions without a hair were generally dome-shaped, brown-red papules with a central black keratinous plug surrounded by a whitish scale (fig. 1b). There were also parafollicular lesions in the left malleolar area.

Histological analysis of a skin punch biopsy from the right leg revealed characteristics more consistent with KD than FD: epidermal invagination of orthokeratotic and parakeratotic central plugs containing basophilic debris (fig. 1c). On the other hand, the patient also exhibited the shallow, nonpenetrating central plugs typical of FD, rather than the large, deep penetrating plugs typical of KD (fig 1c). Therefore, based on the combination of FD and KD features, the patient was diagnosed with KD-FD, despite the small size of the lesions. We treated the patient with oral isotretinoin $(10 \mathrm{mg} / \mathrm{kg})$ for 3 months. Although she showed marked improvement within the second week of treatment, she unfortunately relapsed 3 weeks after discontinuing treatment.

\section{Discussion}

FD and KD generally occur separately in patients between 30 and 50 years of age (emedicine.medscape.com/article/1074469). The present study describes a rare case of 
Alshami and Mohana: A Case of Infantile Kyrle-Flegel Disease in a 6-Year-Old Yemeni Girl

infantile KD-FD that could have been misdiagnosed as FD owing to the findings of a standard clinical examination. Because the diagnostic criteria are based on clinical reports of adult patients with FD or KD, it is important to determine whether they recognize the skin manifestations in a child with both diseases.

The adult manifestations of KD are multiple, well-demarcated papular lesions of variable sizes $(0.5-2 \mathrm{~cm})$. The lesions mainly occur on the scalp, back, and extensor surface of the legs and are asymmetrically distributed and hyperpigmented with central round or irregularly shaped keratotic plugs [11]. A recent study described two siblings who developed KD at 2 and 4 years of age and were examined at 7 and 10 years of age, respectively. Both presented with generalized, multiple, spontaneously occurring discrete keratotic and centrally umbilicated papules with peripheral scaling on the face, limbs, and trunk [5]. In another report of infantile KD, a 7-year-old girl presented with multiple, well-defined, discrete hyperpigmented keratotic papulonodular lesions with a crusted crateriform center on the extensor surface of the upper and lower extremities [3]. The present case had the histological characteristics of KD and the clinical characteristics of FD. These findings are consistent with an earlier report of three siblings (7-13 years of age) with KD-FD, except that the lesions in our case involved the face, were not at the site of trauma, and did not show a linear pattern [4].

During our workup, we excluded 3 other primary perforating dermatoses that can be confused with KD. The first is perforating folliculitis, in which the epidermal invagination is close to a vellus hair, which was not observed in our case. The second is elastosis perforans serpiginosa, which is a genodermatosis of connective tissue. We excluded this condition owing to the absence of thick elastic fibers around the epidermal invaginations, as assessed by elastic Van Gieson's stain. The third is reactive perforating collagenosis, which was excluded because degenerated collagen at the base of the perforation was absent. However, FD may have some clinical and histological characteristics similar to those of KD, although epidermal invagination with deeper penetration of the keratinous plug is mostly seen in KD. Our case had the clinical features of FD but the histological features of KD. Consequently, our diagnosis was KD-FD.

Many treatment options for FD and KD have been reported in the medical literature, including administration of local and systemic retinoids, oral antihistamines, and historically, oral vitamin A (emedicine.medscape.com/article/1074469) [4]. We treated the patient in our report with oral isotretinoin $(10 \mathrm{mg} / \mathrm{kg})$ for 3 months. Although she showed marked improvement within the second week of treatment, she unfortunately relapsed 3 weeks after discontinuing treatment.

In conclusion, the presence of an underlying metabolic disorder needs to be excluded before diagnosing a case as primary KD-FD. This can be achieved by obtaining a detailed medical history of the patient and performing the relevant laboratory tests. Although familial primary KD-FD is rare, close relatives should be thoroughly screened in all cases of perforating dermatoses. We emphasize the need for proper serial sectioning and biopsy processing for demonstrating the perforation of the keratotic plug, which occurs in KD but not in FD. Our case supports the hypothesis that KD and FD are different manifestations or at least represent different stages of the same disease.

\section{Statement of Ethics}

Informed consent for publishing the case was obtained from the parents of the patient. 
Alshami and Mohana: A Case of Infantile Kyrle-Flegel Disease in a 6-Year-Old Yemeni Girl

\section{Disclosure Statement}

The authors have no conflicts of interest.

\section{References}

1 Kyrle J: Hyperkeratosis follicularis et parafollicularis in cutem penetrans. Arch Derm Syphilol 1916;123:466-493.

-2 Abele DC, Dobson RL: Hyperkeratosis penetrans (Kyrle's disease). Report of a case in a Negro with autopsy findings. Arch Dermatol 1961;83:277-283.

-3 Azad K, Hajirnis K, Sawant S, Sabnis V: Kyrle’s disease. Indian Dermatol Online J 2013;4:378-379.

4 Singh S, Bumb RA: Kyrle-Flegel disease in siblings with childhood onset and Koebner phenomenon. Indian J Dermatol Venereol Leprol 1993;59:22-23.

-5 Viswanathan S, Narurkar SD, Rajpal A, Nagpur NG, Avasare SS: Rare presentation of Kyrle's disease in siblings. Indian J Dermatol 2008;53:85-87.

6 Flegel H: Hyperkeratosis lenticularis perstans. Hautarzt 1958;9:363-364.

7 English JS, Hunter I, Skerrow CJ, Skerrow D, Finlay AY: Kyrle-Flegel's - one or two diseases? J R Soc Med 1984;77:16-18.

-8 Squier CA, Eady RA, Hopps RM: The permeability of epidermis lacking normal membrane-coating granules: an ultrastructural tracer study of Kyrle-Flegel disease. J Invest Dermatol 1978;70:361-364.

-9 Bean SF: The genetics of hyperkeratosis lenticularis perstans. Arch Dermatol 1972;106:72.

10 Kocsard E, Palmer G, Constance TJ: Coexistence of hyperkeratosis lenticularis perstans (Flegel) and hyperkeratosis follicularis et parafollicularis in cutem penetrans (Kyrle) in a patient. Acta Derm Venereol 1970;50:385-390.

11 Swetha C, Sathweek RS, Srinivas B, Unnikrishnan R, Anjana RM, Mohan V: A case of Kyrle's disease with diabetes and renal insufficiency. J Diabetol 2014;2:6. 

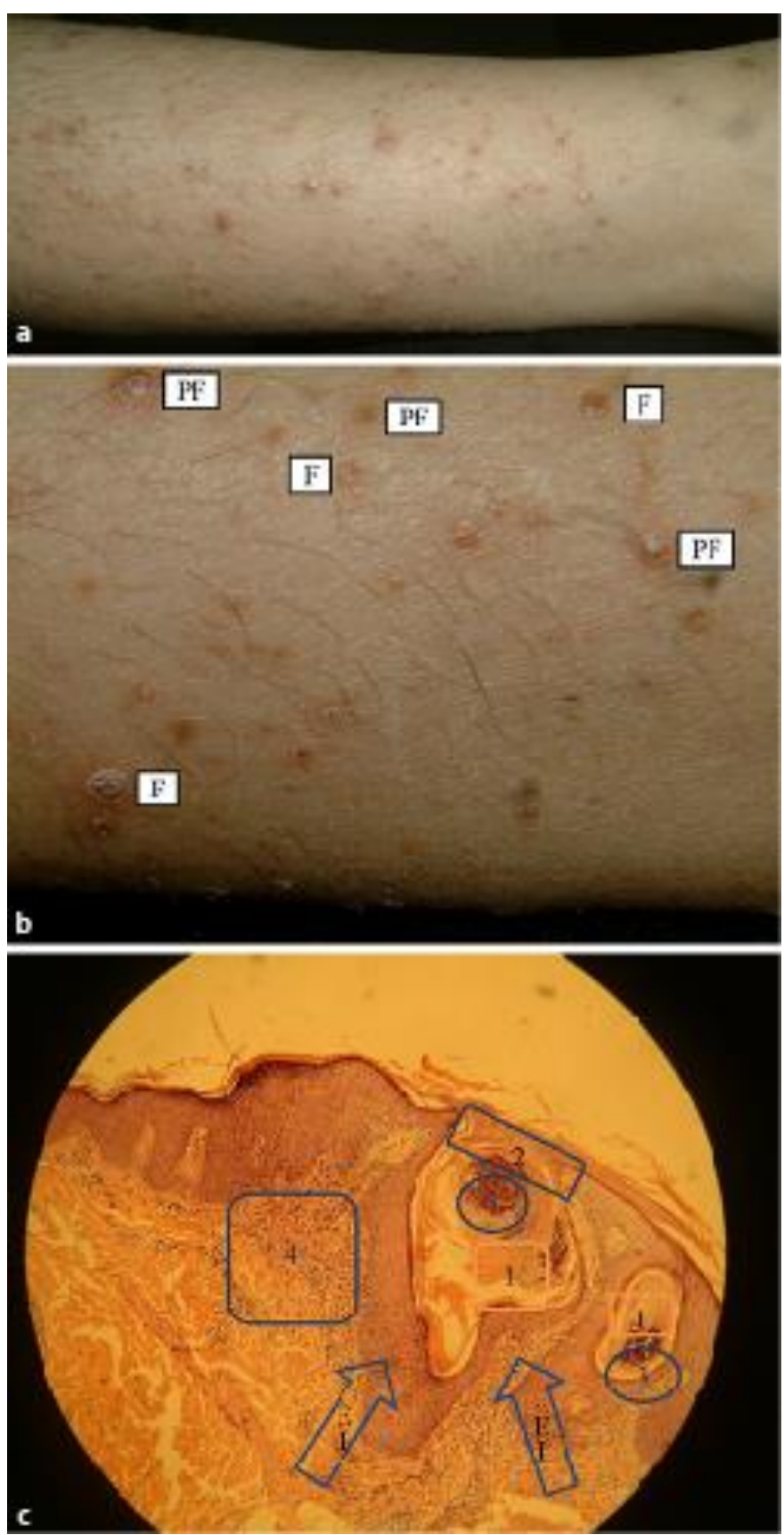

Fig. 1. a Lateral view of the right leg, showing multiple small red-brown papules $1-3 \mathrm{~mm}$ in size with a central keratotic plug. b Close-up view of the area displayed in a, showing many typical lesions at different stages of development. A hair can be seen protruding out of some of the follicular (F) lesions but not out of the parafollicular (PF) lesions. c Histology of the lesion, showing the keratinous plug (1) filling the epidermal invagination (EI). Parakeratosis (2) and basophilic debris (3) can be seen within the plug. There is a dense inflammatory infiltrate (4) in the upper dermis. 